\title{
Organic Affective Syndrome
}

National Cancer Institute

\section{Source}

National Cancer Institute. Organic Affective Syndrome. NCI Thesaurus. Code C34871.

A mental disorder caused by intrinsic disease which is characterized by persistent alterations in mood that are directly attributable to the disease itself rather than a response to knowledge of the disease or other co-morbidity. It may be broadly classified as a depressive or bipolar disorder due to a general medical condition. 